\title{
The dynamic wound microbiome
}

\author{
Chunan Liu ${ }^{1,2}$, Alise J. Ponsero ${ }^{1,2}$, David G. Armstrong ${ }^{3}$, Benjamin A. Lipsky ${ }^{4,5}$ and Bonnie L. Hurwitz ${ }^{1,2^{*}}$
}

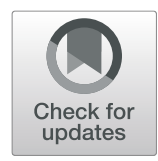

\begin{abstract}
Background: Diabetic foot ulcers (DFUs) account for the majority of all limb amputations and hospitalizations due to diabetes complications. With 30 million cases of diabetes in the USA and 500,000 new diagnoses each year, DFUs are a growing health problem. Diabetes patients with limb amputations have high postoperative mortality, a high rate of secondary amputation, prolonged inpatient hospital stays, and a high incidence of re-hospitalization. DFU-associated amputations constitute a significant burden on healthcare resources that cost more than 10 billion dollars per year. Currently, there is no way to identify wounds that will heal versus those that will become severely infected and require amputation.

Main body: Accurate identification of causative pathogens in diabetic foot ulcers is a critical component of effective treatment. Compared to traditional culture-based methods, advanced sequencing technologies provide more comprehensive and unbiased profiling on wound microbiome with a higher taxonomic resolution, as well as functional annotation such as virulence and antibiotic resistance. In this review, we summarize the latest developments in defining the microbiology of diabetic foot ulcers that have been unveiled by sequencing technologies and discuss both the future promises and current limitations of these approaches. In particular, we highlight the temporal patterns and system dynamics in the diabetic foot microbiome monitored and measured during wound progression and medical intervention, and explore the feasibility of molecular diagnostics in clinics.

Conclusion: Molecular tests conducted during weekly office visits to clean and examine DFUs would allow clinicians to offer personalized treatment and antibiotic therapy. Personalized wound management could reduce healthcare costs, improve quality of life for patients, and recoup lost productivity that is important not only to the patient, but also to healthcare payers and providers. These efforts could also improve antibiotic stewardship and control the rise of "superbugs" vital to global health.
\end{abstract}

Keywords: Diabetic foot ulcer, Wound microbiome, Metagenomics, Next-generation sequencing

\section{Background}

Chronic wounds are a common complication of diabetes mellitus that can severely affect a patient's quality of life and may lead to lower limb amputation [1-5]. The 5-year mortality for diabetic foot ulcers (DFUs), and minor and major amputations was recently reported to be $30.5,46.2$, and $56.6 \%$ [6]. In particular, foot ulcers are prevalent in patients with

\footnotetext{
* Correspondence: bhurwitz@email.arizona.edu

'Department of Biosystems Engineering, University of Arizona, Tucson, AZ, USA

${ }^{2} \mathrm{BIO} 5$ Institute, University of Arizona, Tucson, AZ, USA

Full list of author information is available at the end of the article
}

long-standing diabetes, mostly related to peripheral neuropathy and ischemia from peripheral vascular disease [7]. These DFUs are always colonized by microorganisms and often become infected by pathogenic microbes, resulting in a diabetic foot infection (DFI). It is estimated that $44-68 \%$ of patients admitted to the hospital with a DFI will develop diabetic foot osteomyelitis (DFO) which often requires surgical treatments and a long antibiotic therapy [8-10]. Further, biofilm-producing microbes delay healing and underlie up to $90 \%$ of chronic wounds [11]. Detecting when a DFU is infected is often difficult due to a lack of local or systemic signs or symptoms, primarily

(c) The Author(s). 2020 Open Access This article is licensed under a Creative Commons Attribution 4.0 International License, which permits use, sharing, adaptation, distribution and reproduction in any medium or format, as long as you give appropriate credit to the original author(s) and the source, provide a link to the Creative Commons licence, and indicate if changes were made. The images or other third party material in this article are included in the article's Creative Commons licence, unless indicated otherwise in a credit line to the material. If material is not included in the article's Creative Commons licence and your intended use is not permitted by statutory regulation or exceeds the permitted use, you will need to obtain permission directly from the copyright holder. To view a copy of this licence, visit http://creativecommons.org/licenses/by/4.0/. The Creative Commons Public Domain Dedication waiver (http://creativecommons.org/publicdomain/zero/1.0/) applies to the data made available in this article, unless otherwise stated in a credit line to the data. 
related to the perturbed neurological, vascular, and local inflammatory responses in these patients [12-15]. The standard approach for identifying microorganisms colonizing a wound is to obtain a specimen (preferably of tissue or bone) for aerobic and anaerobic culture. However, achieving results with this time-honored approach usually takes several days (up until 14 days) and is biased toward a subset of microbes that grow well in the laboratory setting. Furthermore, the likelihood of false-negative cultures increases when patients are treated with antimicrobials or clinicians employ inadequate sampling methods, especially superficial swabs of open wounds. False positives can result due to an inability in distinguishing pathogens from healthy skin flora or contaminants. Culture-based approaches have also been found to fail to identify most fungi and frequently do not accurately represent the complete bacterial communities present in the wound [16]. Hence, treatment based on the results of standard culture may fail to cover one or more important pathogens in a DFI [17].

These deficiencies of standard culture have led researchers to seek more technologically advanced methods to identify pathogens from infected wounds. Recently, newer sequencing methods have better defined the surprising microbial diversity found in many areas of the human body ("human microbiome") and the dynamic status of these microorganisms [18-20]. These technologies have provided extensive descriptions of the microbiomes of the gastrointestinal tract and, more recently, the skin [21]. A better understanding of the skin microbiome, which generally holds the organisms responsible for DFIs, should advance our diagnostic and therapeutic approaches to DFIs. Moreover, the characterization of the microbiome associated with osteomyelitis of diabetic foot is also critical as the bone can harbor a bacterial community distinct from the skin. The microbiological spectrum of foot osteomyelitis in diabetic patients is similar to that of the contiguous softtissue infection but fewer numbers of isolates are usually found in bone [8]. Sequencing technologies have detected significantly more anaerobes and Gram-positive bacilli in bone samples compared to conventional techniques, which may contribute to the poor success rate of medical treatment of DFO [9]. Thus, we herein review the latest developments in defining DFU microbiology that has been unveiled by sequencing technologies and discuss both the future promises and current limitations of these approaches. Moreover, we focus on how these technologies can be applied to monitoring and measuring wound progression.

\section{Molecular techniques and limitations}

Thanks to increasingly affordable sequencing platforms and the development of rapid and efficient bioinformatics tools, there have been an increasing number of cultureindependent (molecular) studies for characterizing various human microbiomes (summarized in Table 1). Compared to culture-based techniques, DNA sequencing of the small subunit ribosomal RNA (SSU rRNA) gene and metagenomic next-generation sequencing (mNGS) provide a comprehensive and precise description of the microbial community. These studies have demonstrated that the vast majority of microorganisms in these microbiomes are not detected by standard culture methods, while molecular techniques allow the characterization of microbial populations in their environment using the genetic content of the entire community [22]. Importantly, culturomics approaches have been developed to address the limitations of classical culture methods to increase throughput and allow for the identification of unknown bacteria through $16 \mathrm{~S}$ rRNA sequencing as reviewed in Lagier et al. [23]. Culturomics has been successfully applied to several human-associated microbial communities, and Jneid et al. [24] have confirmed its complementary role in relation to molecular methods in the exploration of complex microbiota in DFIs.

\section{Amplicon sequencing}

Most studies of the microbiology of DFU are based on the amplification of the small subunit ribosomal RNA (SSU rRNA; 16S rRNA for bacteria and archaea, 18S rRNA for eukarya). The SSU rRNA gene is highly conserved; however, variations in hypervariable regions can be used to distinguish microbial species. Molecular fingerprinting methods targeting the SSU rRNA gene, such as denatured gradient gel electrophoresis (DGGE) or a temperature gradient gel electrophoresis (TGGE), are frequently used to rapidly identify microbes and monitor differences in composition over space or time [25]. These methods separate DNA fragments of equal length based on their sequence melting point. Therefore, compositional diversity can be rapidly visualized using these methods where each band on a gel represents a specific prokaryotic taxon, although in certain cases, two different species can migrate at the same length, leading to possible misidentifications. Although DGGE and TGGE can be used for better characterization of complex microbial flora than culture-based methods, the identification of the specific prokaryotic species present in the sample can be difficult.

Amplicon sequencing allows for the identification and characterization of microbial diversity and to identify microbial community members using amplification, sequencing, and analysis of the SSU rRNA gene. Older studies relied on Sanger sequencing methods to obtain full-length SSU rRNA gene sequences, but because of the cost and sequencing capacity, only for a small subset of the organisms in a sample. Next-generation sequencing (NGS) 


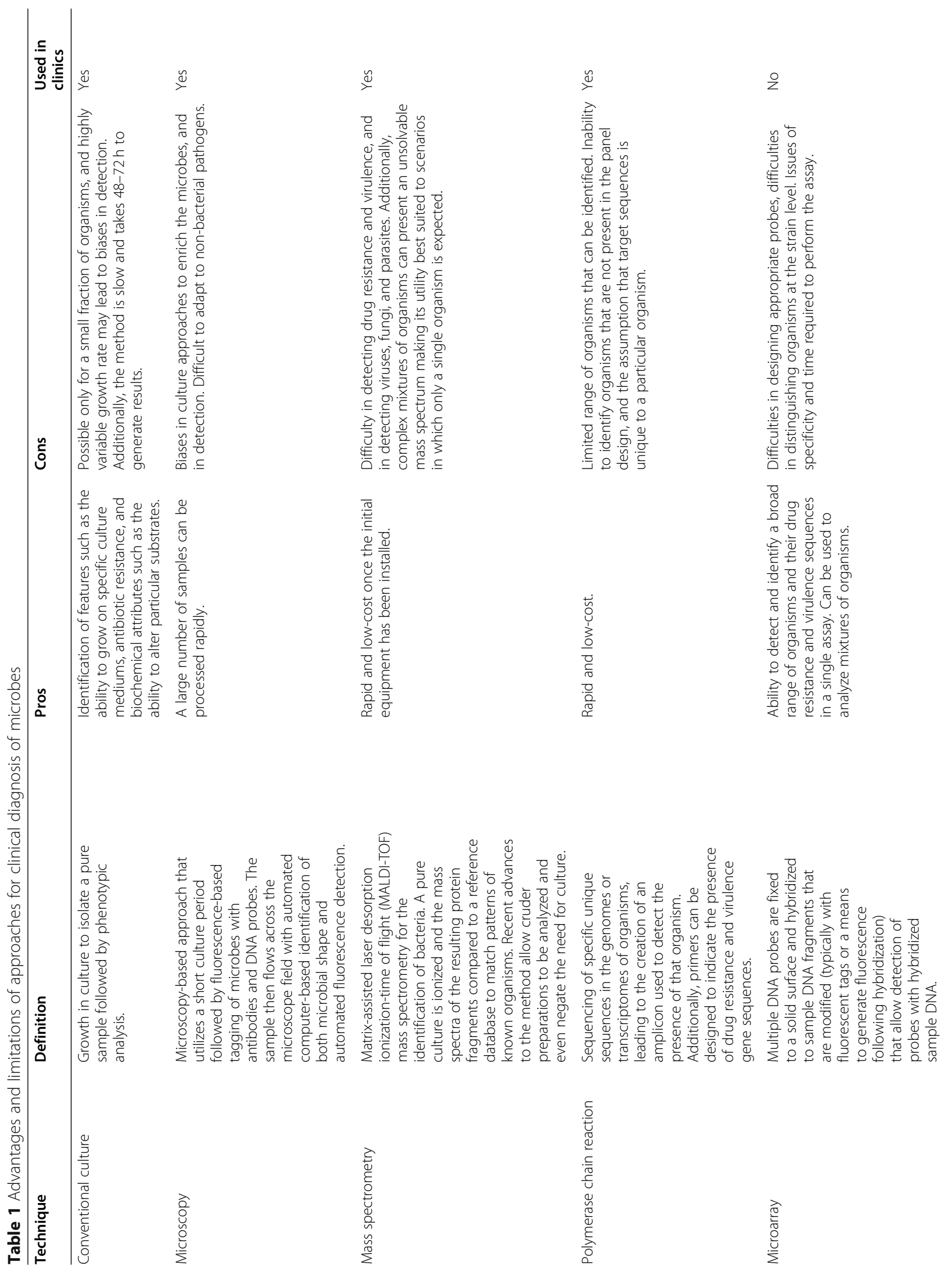




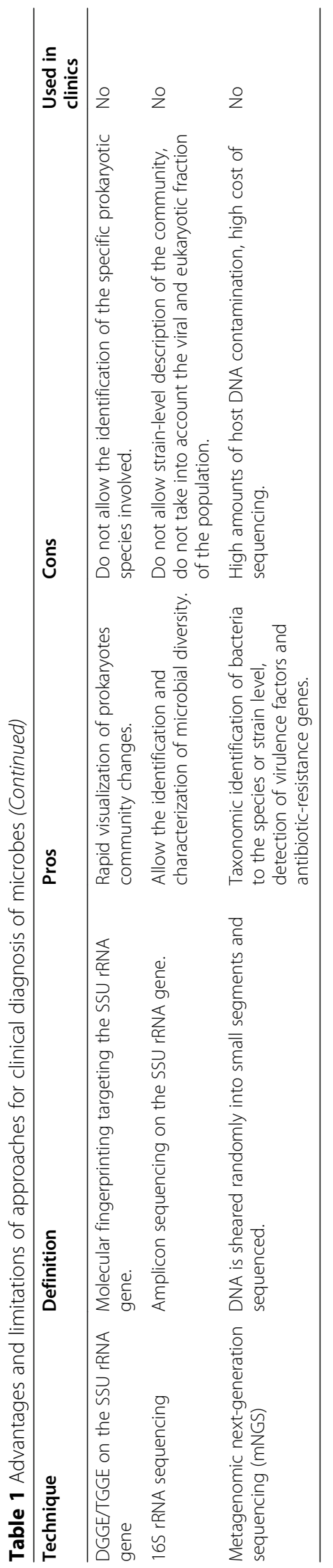


techniques have vastly improved sequencing depth, but have shorter $(\sim 500 \mathrm{bp})$ sequences and therefore typically use one or more hypervariable regions as a proxy for the full-length gene.

Amplicon-based studies on DFU samples reveal a much more complex bacterial community associated with DFU than those identified by culture $[26,27]$ and provide some insight into patient outcomes for these wounds $[28,29]$. However, these approaches are limited by amplification biases, as no universal SSU rRNA primers exist, and the primer choice affects the amplification efficiency of different microbial phyla. Furthermore, the quality of DNA extraction varies according to the microbial taxa [30]. Importantly, these methods are restricted to prokaryotes and fungi and do not account for bacteriophages and eukaryotic viruses, which have been shown to modulate virulence and biofilm formation of Staphylococcus aureus in DFU [31]. While 16S rRNA amplicon studies allow for the identification of microbes down to the genus level, these approaches cannot be used for species- or strain-specific characterization. While bioinformatics tools now exist to predict functional content from $16 \mathrm{~S}$ sequences [32], these methods have been shown to be biased toward known microbial groups and pathways [30]. Additionally, these predictive analyses cannot infer antibiotic resistance and virulence genes that are of interest in clinical samples.

\section{Metagenomic next-generation sequencing}

Despite a growing number of studies using sequencing to investigate microbial communities in wounds, few use metagenomic next-generation sequencing (mNGS) approaches (mNGS workflow is shown in Fig. 1). Unlike amplicon-based sequencing methods, mNGS indiscriminately sequences all genes for all organisms in a given microbial population at their relative abundance in the sample, including viruses and other mobile elements [33]. When compared with 16S-based metagenomics, it offers a finer taxonomic identification for bacteria to the species or strain level. In their 2019 study, Kalan et al. compared 16S rRNA amplicon sequencing and mNGS results for 195 samples from 46 DFU patients [34]. Overall, each approach identified the same major bacterial genera and was significantly correlated based on taxonomic diversity in the samples. However, mNGS offered additional insights into the strain-level diversity of $S$. aureus, bacteria that have been shown to drive outcomes in patients with a DFU [34]. Moreover, mNGS allows an estimation of metabolic pathways present in the microbial population. In chronic wounds, identifying virulence factors and antibiotic-resistance markers in the microbial community can be particularly helpful in selecting antimicrobial therapy. Using mNGS, Kalan and collaborators showed that an increase in genes related to biofilm formation in the microbial population was associated with non-healing DFU [34].

In conclusion, mNGS avoids amplification bias, captures both cellular and viral components of the community, and allows for species/strain-level analysis. These datasets are, however, more expensive to generate and require greater computational knowledge and resources to store, process, and analyze. Moreover, humanassociated microbiomes often contain a large amount of human DNA contamination. This is especially problematic when sampling wound tissues and bone specimens. The vast majority of reads are human in unenriched samples [35], due to human contamination from lysed human cells and free cellular DNA. Thus, samples either need to be enriched, leading to low quantities of DNA and the potential to sequence contaminants or deeply sequenced, leading to extensive costs that greatly exceed the cost for culture.

Finally, although both amplicon-based and mNGS approaches allow the identification of microorganisms in an environmental sample, unlike cultures, they do not distinguish living from dead or dormant microorganisms. For this reason, and because many chronic wound samples reveal multiple isolates, it is particularly challenging to identify which microbes are actively contributing to infection in this setting.

\section{Different approaches may reveal different microbial populations}

Culture-based approaches for the identification and quantitation of microbes in wounds are limited by the fact that various factors impair their ability to identify the causative microorganisms that are both viable and actively infecting (rather than colonizing or contaminating) the wound. A first step in obtaining accurate results is to obtain high-quality samples to submit for culture. This begins with proper cleaning and debriding the wound (mainly performed by a specialized surgery in the operating room), then having specimens collected by an appropriately trained clinician supplied with proper equipment, taking tissue curetting or biopsies (not swabs), and ensuring the time and care to avoid procedures that might contaminate the specimen. To ensure the microorganisms in the specimen remain viable uncontaminated and viable, they must be carefully handled and quickly delivered to the microbiology lab for culturing using adapted transport methods and media. In the laboratory, specimens must be inoculated on the proper media, under appropriate conditions, and for the required duration to allow growth (especially if seeking anaerobic prokaryotes). Oftentimes, culture results identify and report organisms that easily grow in the laboratory environment, but that underestimates the diversity of 


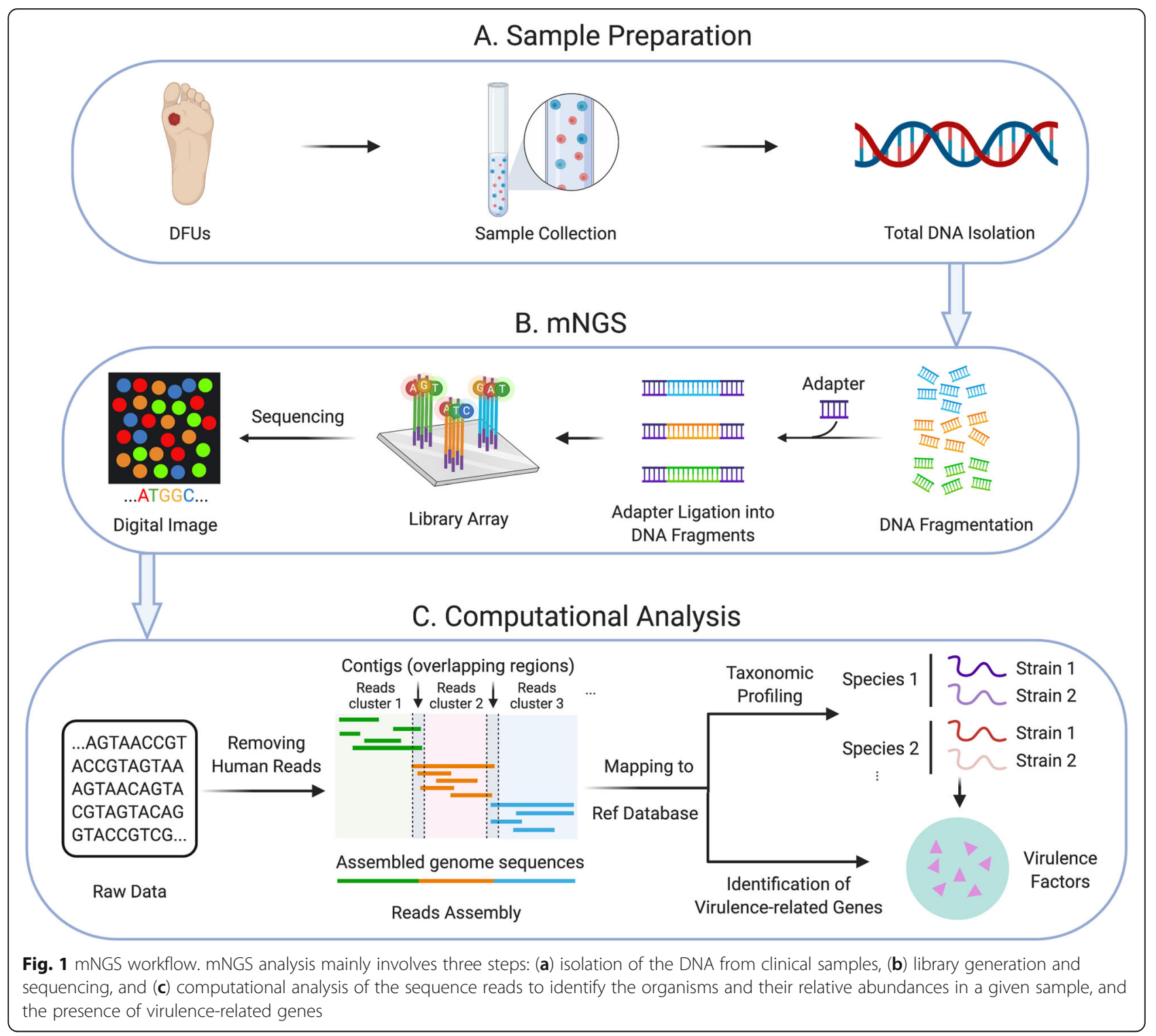

the skin microbiota in samples from both healthy hosts and those with a chronic wound [16, 36]. Obligately, anaerobic prokaryotes are often present in DFUs, but they are often fastidious and are only detectable impeccable culture methods or molecular sequencing approaches [19]. Thus, cultures may have a high false-negative rate and lack full representation of the complete microbial population in wounds.

With microbiome surveys becoming more prevalent in studies of patients with chronic wounds or DFUs, it is important to compare the techniques the authors used and assess their limitations. In a 2016 study, Meisel et al. compared the effect of sequencing different $16 \mathrm{~S}$ hypervariable regions for studying skin microbiota samples. Their results suggest that the V1-V3 region provides a more accurate taxonomic assessment than the $\mathrm{V} 4$ region
[37]. Moreover, the authors compared the genetic predictions based on $16 \mathrm{~S}$ rRNA sequencing and pathways annotations from mNGS datasets. The two approaches revealed similar results, suggesting that in some instances genetic prediction can provide a reasonable estimate of functional enrichment when mNGS cannot be performed.

\section{The dynamic DFU microbiome}

\section{The diabetic skin microbiome}

The skin microbiome is not only a complex (containing multiple species) but a highly dynamic microbial community that maintains an interdependent relationship with the human host. In a healthy state, skin commensal microbes co-exist with their human hosts and help prevent potential pathogens from colonizing the skin. In 
response to skin damage, the host immune system triggers an inflammatory cascade to help avert pathogen invasion and initiate the healing process. Some bacteria residing on the skin can exert antimicrobial activities against pathogens [38] or decrease the pathogen virulence [39] to protect the host. Importantly, commensal microbes can often be distinguished from pathogens based on pathogen-associated molecular patterns (PAMPs) or molecules that are associated with infectious agents in the affected tissue $[40,41]$. That said, organisms that are often considered non-pathogens can cause infection in hosts with impaired immunity or who have recently received antimicrobial treatment.

When immune responses are impaired, as is often the case of persons with diabetes, they may fail to prevent colonization by pathogenic bacteria in the wounded tissue. In chronically infected wounds, many bacteria form a biofilm, in which they irreversibly attach to and grow on a surface, produce extracellular polymers that facilitate matrix formation, and alter their phenotype with respect to growth rate and gene transcription.

Of note, studies have demonstrated clinically relevant differences in the foot's skin microbiome between nondiabetic and diabetic individuals, which likely contribute to the higher rates of skin-associated infectious diseases in diabetic patients [42]. A recent longitudinal study [43] followed patients with uninfected DFUs over a period of 10 weeks, comparing the microbial population of the DFU to the skin on the contralateral site on the same patient, as well as a control group of healthy subjects without diabetes. They found that the microbiome of the wound was different from the unaffected skin in the diabetic patients (contralateral site to the wound), and notably, the microbiome of the unaffected skin was significantly less diverse in these diabetic persons than that in non-diabetic persons. They also identified 69 operational taxonomic units (OTUs) specific to the diabetic skin microbiome that may provide further insight into variations in healing.

\section{DFU microbial composition and patient outcomes}

Predictive analytics (using data, statistical algorithms, and machine learning techniques to identify the likelihood of future outcomes based on historical data) can help to guide treatment by identifying patterns in microbial species composition linked to patient outcomes. Several recent studies have begun to explore differentiating the microbial composition of healing and chronic wounds. In one, infected DFUs with a long duration $(\geq$ 6 weeks) were found to have a more diverse microbiome than rapidly healing ones [44]. These severely infected DFUs had altered microbial community structures compared to mild or moderate infections, showing a stratification between wounds and their severity. Moreover, results from MacDonald et al. [45] suggested that healing DFUs had a higher abundance of Actinomycetales and Staphylococcaceae, while non-healing DFUs showed higher abundances of Bacteroidales and Streptococcaceae. Facultative anaerobes, especially of the genus Enterobacter, were found to be significantly associated with lack of healing, and thus a negative prognostic factor derived from knowing the chronic wound microbiome [46]. However, despite these trends, no statistically significant correlation was found between wound status (healed versus unhealed) and the abundance of any particular taxa at the species or genus level in several other studies [29, 43, 47].

Bacterial strain-level variations can also have important functional differences that influence their interactions with their host. Staphylococci isolated from DFUs have been found to be genetically diverse [48], among which $S$. aureus is a major colonizer that produces abundant biofilm and thereby inhibits wound healing resulting in wound infections $[49,50]$. Kalan and colleagues found that for DFU, using mNGS showed that variations in strains of $S$. aureus were correlated with ulcer healing time [34]. Importantly, strain-level variation of S. aureus genes was also predictive of poor outcomes in a type 2 diabetes mouse model [34].

The occurrence of pathogenic and multidrug-resistant strains like methicillin-resistant $S$. aureus (MRSA) strains negatively influences treatment outcomes and leads to chronicity of ulcers $[49,51,52]$. Shettigar and Murali [52] recently grouped strains of $S$. aureus into clonal lineages, along with their associated virulence markers involved in skin and wound infection to help in distinguishing between colonizing and pathogenic strains. Using mNGS, determining the presence of genes encoding antibiotic resistance, and virulence factors can help in better understanding and predicting patient outcomes. Kalan et al. [34] showed that antibiotic-resistance genes are abundant in microorganisms comprising the DFU microbiome. In particular, the authors isolated a complete genome for $S$. aureus that carried multiple antibiotic-resistance genes and found it was most abundant in samples from patients with poor outcomes. Slow-healing and chronic wounds were also enriched with organisms in biofilm-related pathways, including the agr $A B C D$ operon that encodes genes important for biofilm development and virulence [34]. Likewise, Sloan et al. [47] identified genes for tetracycline and macrolide resistance in strains of Streptococcus anginosus and Prevotella intermedia from DFU samples. However, neither tetracyclines nor macrolides are currently commonly used by clinicians for treating diabetic foot infections, except in selected scenarios, e.g., for patients who are penicillin-allergic or for infections with pathogens that are resistant to other commonly used antibiotic agents. 
Due to the polymicrobial nature of DFU, understanding the complex interactions between pathogens and the commensal flora, rather than the simple presence or absence of specific bacteria, is often more informative with respect to the evolution of the wound [53]. For example, the pathogenic effects of anaerobes can be increased by the presence of aerobes as they consume oxygen inducing tissue hypoxia, and facilitating the growth of anaerobes [54,55]. Such symbiotic relationships are defined as the cooperative interaction of two or more species that may result in an increase in virulence leading to delayed healing [56]. Pseudomonas aeruginosa and S. aureus, the two most common causes of chronic wound infections, are frequently found together, and combined $P$. aeruginosa and $S$. aureus infections are more virulent than single infections [57-60]. Additionally, a wound model has shown that when grown in culture together, $P$. aeruginosa and $S$. aureus display an enhanced antibiotic tolerance [60]. Bacteroides fragilis has been reported in several studies as the predominant anaerobic bacteria isolated in DFIs [61-63], and is also important in community dynamics and biological interactions. Mastropaolo et al. [64] demonstrated the effect of polymicrobial infection involving Escherichia coli, Bacteroides fragilis, and Clostridium perfringens in a mouse model of type 2 diabetes.

Aside from synergistic interactions between pathogens, competitive interaction between non-pathogenic commensal bacteria and pathogens has been observed in wounds. Indeed, the presence of the commensal Helcococcus kunzii in wounds significantly reduced the virulence of the $S$. aureus without directly modifying the host defense response [39]. In addition, prophage inserted into the $S$. aureus host genome in a DFU also appears to attenuate bacterial virulence [31]. All in all, there is growing evidence that polymicrobial interactions may synergize the pathogenic potential or decrease the virulence of other microorganisms and have a major impact on the severity and evolution of wound infection. Therefore, it is of great importance to move beyond the presence/absence survey of bacteria in DFUs and exploring potential microbial interactions in wounds.

\section{Effect of medical intervention on the DFU microbiome}

Effective therapeutic interventions are critical to the management of a DFU to prevent them from becoming infected or chronic, which can lead to many adverse consequences, including lower extremity amputation. Required treatments generally include adequate glycemic control as well as the debridement of compromised or necrotic tissues, offloading pressure, covering wounds with appropriate dressings, and for clinically infected wounds, administering appropriate antimicrobial therapy
[65]. It is also useful to monitoring the evolution of the DFU microbiome in response to treatment to help ensure that (1) new pathogens have not emerged that are not covered by the current antibiotic treatment; (2) the antimicrobial therapy is the narrowest spectrum needed, in concordance with the principles of antibiotic stewardship [66]; and (3) the target organisms are being eradicated with the current treatment.

Of course, antibiotic treatment itself may drive major changes in wound microbiota composition $[16,45,67$, 68]. Sloan et al. [47] found a reduction in microbial diversity in DFU wounds after affected patients had been treated with doxycycline, ciprofloxacin, and metronidazole. This reduction in diversity can potentially lead to the unchecked growth of pathogens in the wound. For example, empirical treatment with doxycycline in a DFU was followed later by an expansion of streptococci, while in another wound a subsequent expansion of Enterobacteriaceae was observed following treatment with coamoxiclav to an episode of clinical infection caused by Anaerococcus and Peptonophilus [47]. Other studies report contradictory evidence, where DFUs in patients with exposure to systemic antibiotics who demonstrated delayed healing remained stagnant and did not show any significant changes in terms of microbial community diversity or composition [29,34], compared to those that healed. Loesche and colleagues propose that wounds should optimally be dynamic during the healing process, where the microbiome shifts from infected to normal skin flora [29].

Wound debridement and wound dressings have also been shown to significantly modify the wound microbiome in DFU patients, with evidence that this led to favorable outcomes [34], even for long-term chronic wounds [43]. However, Verbanic et al. [46] found no difference in the wound microbiome between predebridement and 1-2 min post-debridement specimens, suggesting that debridement did not alter the wound microbiome directly in the short term. Notably, in this study, the extent and depth of debridement, as well as the type of instrument used (curette, scalpel, scissors, or tissue nipper), were not standardized and were determined by the treating physician. Additionally, abrupt changes in DFU community composition have also been reported without any obvious causes, such as antibiotic exposure or the development of clinical infections [47]. Other treatments like probiotic bacteria and phage therapy are potential alternatives that may have effects on the DFU microbiome, but have not yet been welldefined [69-71].

Finally, the effect of antibiotics may be limited, especially under in light of the increasing problem of antimicrobial drug resistance. When antibiotic-resistant bacteria emerge, they are typically difficult to eradicate, 
contributing to the low efficiency of antibiotic therapy in DFU management [15, 41]. Additionally, many clinics rely on a traditional culture that underestimates wound flora and may lead to inappropriate antibiotics prescribed in up to $45 \%$ of cases [72, 73]. The excessive (too broad-spectrum or of unnecessarily long duration) or inappropriate (treating clinically uninfected wounds) use of antibiotics not only results in ineffective treatment but also aggravates the worldwide crisis of antibiotic resistance. mNGS holds great promise as a method to accurately and quickly detect and quantify genes related to antibiotic resistance, thus assisting clinicians to better select the most appropriate antibiotic regimen. However, long-term adoption and utility of these methods in the clinic may be limited, given the relatively long time to get a result and difficulties in convincing both clinicians and patients to change to a more targeted drug once a course of broad-spectrum antibiotics has been started.

\section{Moving to the clinic}

Despite recent strides in research in the microbiology of DFU, using molecular approaches (16S rRNA and mNGS) to assess the composition of the microbial community, developing a clinical diagnostic algorithm and using these approaches will require first demonstrating both their clinical validity and utility. Specifically, clinical trials must not only validate molecular results compared to culture but also assess the cost-effectiveness of the resulting treatment approaches. In particular, are the added costs for molecular methods justified by providing faster and more comprehensive results that actually improve outcomes, either for the patient or for society as a whole? Can molecular diagnostics prevent hospitalization and amputation in a significant number of patients with wound infections? Further, if mNGS detects antibiotic resistance or virulence genes, are these genes expressed, and is it helpful to use this information to select an optimal antibiotic regimen? Currently, these questions remain unanswered.

\section{Temporal patterns and system dynamics in DFU}

In addition to providing results that validate culture, mNGS approaches also have the potential to provide unbiased information on the abundance of organisms in the entire community. This may result in recognizing that knowing which combinations of organisms are present and their relative abundance in the community are associated with improved patient outcomes and the clinical or environmental factors that drive these differences. These complex interactions between microbes, the human host, and the skin environment are key to holistically understanding wound ecology. Yet, capturing the richness of these community dynamics requires temporal sampling to capture the full richness of community interactions. It will also be important to determine if, and how often, wounds should be sampled to determine the microbiome present. The frequency of sampling should probably match the rate of change of the system in order to reveal characteristic fluctuations in microbial communities based on factors that are clinically or environmentally cogent.

To date, most studies of patients with a DFU are limited by both cohort size and sample collection methods and frequency, thus preventing the detection of dynamic dependencies in community composition. In particular, small cohort studies lack statistical robustness and cannot be used worldwide by adjusting for geographic differences [41, 74]. Thus, developing methods to measure and monitor microbial communities in DFU over time to detect their association with patterns of healing may require careful data pooling and machine learning techniques. These techniques could, however, be useful if detecting certain patterns provides clinically relevant early warning signs in wound ecology. These computational methods could provide a deeper understanding of the microbial dynamics in DFU; this, combined with properly applied predictive analytics, could promote targeted and evidence-based therapeutics and patient care with better outcomes.

Before being recommended for widespread clinical use, molecular diagnostics must be carefully validated in the lab to test the limits of detection of microbes, to ensure low rates of false positive or negative findings, and to monitor for contaminants in reagents or poorly obtained or processed microbial samples [35]. Moreover, most studies lack control subjects to ensure any detected differences in microbial community composition and dysbiotic states in ulcers are accurate. To our knowledge, the study by Gardiner et al. is the only one that employed a control group, and they had only eight control subjects [43]. Similarly, there is currently no standard protocol for processing samples and consistently analyzing them. As such, results from relevant studies are inconsistent, and the differences in microbial diversity and composition they demonstrate may be due to biases rather than true biological differences. For example, factors such as patient demographics, clinical characteristics, sampling and sequencing protocols, and downstream analytical methods vary widely across studies. Most importantly, metagenomic studies rarely report the types of wounds and treatment characteristics. In particular, knowing about the performance of wound debridement, the grade of the wound, and the depth of infection is critical to allow comparisons and metaanalyses of these studies.

Moving molecular diagnostics into the clinic will require first building a standard workflow to translate 
these advanced techniques into corroborated clinical evidence. Currently, reports from mNGS datasets contain diverse microbes that are not typically observed in culture-based analyses, but we do not know which are clinically relevant. Additionally, virulence factors and antibiotic resistance genes are detected but not quantified based on gene expression levels, making their relevance to clinical decisions also unclear. Lastly, because most physicians are not trained to work with or understand mNGS data, there is a major barrier in interpreting the complex and often contradictory results they provide when it comes to choosing antibiotic therapy. These technologically advanced mNGS methods have given us a broader and more accurate view of the microbiome of wounds. However, it is still difficult to interpret the meaning of these data. Thus, as the most recent guidelines for managing diabetic foot infections suggest [65], we will need further research and refinement before moving into the clinic.

\section{Conclusions}

Diabetic foot ulcers are one of the most common, costly, and severe complications of diabetes that have been regarded as a major public health problem. Accurate identification of causative pathogens is essential for early diagnosis and proper treatment. In this review, we have highlighted the latest developments in defining the microbiology of DFUs unveiled by sequencing technologies with an emphasis on temporal analysis and system dynamics, and discuss both the future promises and current limitations of these approaches. Metagenomics can enable the detection of majority microorganisms compared to traditional culture-based techniques, particularly in terms of not yet cultivable bacteria. Molecular tests conducted during weekly office visits to clean and examine DFUs would allow clinicians to offer personalized treatment and antibiotic therapy. Personalized wound management could reduce healthcare costs, improve quality of life for patients, and recoup lost productivity that is important not only to the patient, but also to healthcare payers and providers. These efforts could also improve antibiotic stewardship and control the rise of "superbugs" vital to global health. Moreover, other recently developed approaches like culturomics may provide a better assessment of minority flora. Future studies using such complementary tools to molecular methods will provide more comprehensive insights into the diabetic foot microbiome.

\section{Abbreviations}

SSU rRNA: Small subunit ribosomal gene; mNGS: Metagenomic nextgeneration sequencing; DGGE: Denatured gradient gel electrophoresis; TGGE: Temperature gradient gel electrophoresis; OTU: Operational taxonomic unit; DFU: Diabetic foot ulcer; DFO: Diabetic foot osteomyelitis

\section{Acknowledgements}

The authors thank Hurwitz Lab and Bio5 Institute, University of Arizona, for writing assistance, language editing, and funding support.

\section{Authors' contributions \\ All authors contributed to writing this manuscript. All authors read and approved the final manuscript.}

\section{Funding}

This work was supported by the startup fund to Dr. Bonnie Hurwitz from Bio5 Institute, University of Arizona.

\section{Availability of data and materials}

Not applicable.

Ethics approval and consent to participate

Not applicable.

Consent for publication

Not applicable.

\section{Competing interests}

The authors declare that they have no competing interests.

\section{Author details}

${ }^{1}$ Department of Biosystems Engineering, University of Arizona, Tucson, AZ, USA. ${ }^{2} \mathrm{BIO} 5$ Institute, University of Arizona, Tucson, AZ, USA. ${ }^{3}$ Department of Surgery, Southwestern Academic Limb Salvage Alliance (SALSA), Keck School of Medicine of University of Southern California, Los Angeles, USA.

${ }^{4}$ Department of Medicine, University of Washington, Seattle, WA, USA.

${ }^{5}$ Division of Medical Sciences, Green Templeton College, University of Oxford, Oxford, UK.

Received: 13 August 2020 Accepted: 20 October 2020

Published online: 24 November 2020

\section{References}

1. WHO | Global report on diabetes. WHO. http://www.who.int/diabetes/ global-report/en/. Accessed 22 Sept 2020.

2. Armstrong DG, Boulton AJM, Bus SA. Diabetic foot ulcers and their recurrence. 2017. https://doi.org/10.1056/NEJMra1615439.

3. Skrepnek GH, Mills JL, Lavery LA, Armstrong DG. Health care service and outcomes among an estimated 6.7 million ambulatory care diabetic foot cases in the U.S. Diabetes Care. 2017:40:936-42.

4. Lazzarini PA, Pacella RE, Armstrong DG, van Netten JJ. Diabetes-related lower-extremity complications are a leading cause of the global burden of disability. Diabet Med. 2018;35:1297-9.

5. Zhang Y, Lazzarini PA, MCPhail SM, van Netten JJ, Armstrong DG, Pacella RE. Global disability burdens of diabetes-related lower-extremity complications in 1990 and 2016. Diabetes Care. 2020;43:964-74.

6. Armstrong DG, Swerdlow MA, Armstrong AA, Conte MS, Padula WV, Bus SA. Five year mortality and direct costs of care for people with diabetic foot complications are comparable to cancer. J Foot Ankle Res. 2020;13:16.

7. Pendsey SP. Understanding diabetic foot. Int J Diabetes Dev Ctries. 2010;30: 75-9.

8. Hartemann-Heurtier A, Senneville E. Diabetic foot osteomyelitis. Diabetes Metab. 2008:34:87-95.

9. van Asten SAV, La Fontaine J, Peters EJG, Bhavan K, Kim PJ, Lavery LA. The microbiome of diabetic foot osteomyelitis. Eur J Clin Microbiol Infect Dis. 2016;35:293-8.

10. Giurato L, Meloni M, Izzo V, Uccioli L. Osteomyelitis in diabetic foot: a comprehensive overview. World J Diabetes. 2017:8:135-42.

11. Attinger C, Wolcott R. Clinically addressing biofilm in chronic wounds. Adv Wound Care. 2012;1:127-32

12. Armstrong DG, Lipsky BA. Advances in the treatment of diabetic foot infections. https://home.liebertpub.com/dia. 2004. https://doi.org/10.1089/ 152091504773731357

13. Williams DT, Hilton JR, Harding KG. Diagnosing foot infection in diabetes. Clin Infect Dis. 2004;39(Supplement_2):S83-6. 
14. Peters EJG, Lavery LA, Armstrong DG. Diabetic lower extremity infection: influence of physical, psychological, and social factors. J Diabetes Complications. 2005;19:107-12.

15. Lipsky BA, Berendt AR, Cornia PB, Pile JC, Peters EJG, Armstrong DG, et al. 2012 Infectious Diseases Society of America clinical practice guideline for the diagnosis and treatment of diabetic foot infections. Clin Infect Dis. 2012;54:e132-73.

16. Gardner SE, Hillis SL, Heilmann K, Segre JA, Grice EA. The neuropathic diabetic foot ulcer microbiome is associated with clinical factors. Diabetes. 2013:62:923-30

17. Gardner SE, Haleem A, Jao Y-L, Hillis SL, Femino JE, Phisitkul P, et al. Cultures of diabetic foot ulcers without clinical signs of infection do not predict outcomes. Diabetes Care. 2014;37:2693-701.

18. Turnbaugh PJ, Ley RE, Hamady M, Fraser-Liggett CM, Knight R, Gordon J. The Human Microbiome Project. Nature. 2007;449:804-10.

19. Spichler A, Hurwitz BL, Armstrong DG, Lipsky BA. Microbiology of diabetic foot infections: from Louis Pasteur to 'crime scene investigation'. BMC Med. 2015;13:2.

20. Moffarah AS, Mohajer MA, Hurwitz BL, Armstrong DG. Skin and soft tissue infections. Microbiol Spectr. 2016;4. https://doi.org/10.1128/microbiolspec. DMIH2-0014-2015.

21. Byrd AL, Belkaid $Y$, Segre JA. The human skin microbiome. Nat Rev Microbiol. 2018;16:143-55.

22. Vartoukian SR, Palmer RM, Wade WG. Strategies for culture of 'unculturable' bacteria. FEMS Microbiol Lett. 2010;309:1-7.

23. Lagier J-C, Dubourg G, Million M, Cadoret F, Bilen M, Fenollar F, et al. Culturing the human microbiota and culturomics. Nat Rev Microbiol. 2018; 16:540-50.

24. Jneid J, Cassir N, Schuldiner S, Jourdan N, Sotto A, Lavigne J-P, et al. Exploring the microbiota of diabetic foot infections with culturomics. Front Cell Infect Microbiol. 2018;8. https://doi.org/10.3389/fcimb.2018.00282

25. Tzeneva VA, Heilig HGHJ, van Vliet WA, Akkermans ADL, de Vos WM, Smidt H. $16 \mathrm{~S}$ rRNA targeted DGGE fingerprinting of microbial communities. In: Martin CC, Martin CC, editors. Environmental genomics. Totowa: Humana Press; 2008. p. 335-350. https://doi.org/10.1007/978-1-59745-548-0_17.

26. Dowd SE, Wolcott RD, Sun Y, McKeehan T, Smith E, Rhoads D. Polymicrobial nature of chronic diabetic foot ulcer biofilm infections determined using bacterial tag encoded FLX amplicon pyrosequencing (bTEFAP). PLOS ONE. 2008:3:e3326.

27. Dowd SE, Sun Y, Secor PR, Rhoads DD, Wolcott BM, James GA, et al. Survey of bacterial diversity in chronic wounds using pyrosequencing, DGGE, and full ribosome shotgun sequencing. BMC Microbiol. 2008:8:43.

28. Kalan L, Loesche M, Hodkinson BP, Heilmann K, Ruthel G, Gardner SE, et al. Redefining the chronic-wound microbiome: fungal communities are prevalent, dynamic, and associated with delayed healing. mBio. 2016;7. https://doi.org/10.1128/mBio.01058-16.

29. Loesche M, Gardner SE, Kalan L, Horwinski J, Zheng Q, Hodkinson BP, et al Temporal stability in chronic wound microbiota is associated with poor healing. J Invest Dermatol. 2017;137:237-44.

30. McLaren MR, Willis AD, Callahan BJ. Consistent and correctable bias in metagenomic sequencing experiments. eLife. 2019;8:e46923.

31. Messad N, Prajsnar TK, Lina G, O'Callaghan D, Foster SJ, Renshaw SA, et al. Existence of a colonizing Staphylococcus aureus strain isolated in diabetic foot ulcers. Diabetes. 2015;64:2991-5.

32. Langille MGl, Zaneveld J, Caporaso JG, McDonald D, Knights D, Reyes JA, et al. Predictive functional profiling of microbial communities using $16 \mathrm{~S}$ rRNA marker gene sequences. Nat Biotechnol. 2013;31:814-21.

33. Oh J, Byrd AL, Deming C, Conlan S, Kong HH, Segre JA. Biogeography and individuality shape function in the human skin metagenome. Nature. 2014 514:59-64.

34. Kalan LR, Meisel JS, Loesche MA, Horwinski J, Soaita I, Chen X, et al. Strainand species-level variation in the microbiome of diabetic wounds is associated with clinical outcomes and therapeutic efficacy. Cell Host Microbe. 2019;25:641-655.e5.

35. Watts GS, Hurwitz BL. Metagenomic next-generation sequencing in clinical microbiology. Clin Microbiol Newsl. 2020;42:53-9.

36. Findley K, Oh J, Yang J, Conlan S, Deming C, Meyer JA, et al. Topographic diversity of fungal and bacterial communities in human skin. Nature. 2013; 498:367-70.

37. Meisel JS, Hannigan GD, Tyldsley AS, SanMiguel AJ, Hodkinson BP, Zheng Q, et al. Skin microbiome surveys are strongly influenced by experimental design. J Invest Dermatol. 2016;136:947-56.
38. Nakatsuji T, Chen TH, Narala S, Chun KA, Two AM, Yun T, et al. Antimicrobials from human skin commensal bacteria protect against Staphylococcus aureus and are deficient in atopic dermatitis. Sci Transl Med. 2017:9. https://doi.org/10.1126/scitranslmed.aah4680.

39. Ngba Essebe C, Visvikis O, Fines-Guyon M, Vergne A, Cattoir V, Lecoustumier A, et al. Decrease of Staphylococcus aureus virulence by Helcococcus kunzii in a Caenorhabditis elegans model. Front Cell Infect Microbiol. 2017;7. https://doi.org/10.3389/fcimb.2017.00077.

40. Chen L, Deng H, Cui H, Fang J, Zuo Z, Deng J, et al. Inflammatory responses and inflammation-associated diseases in organs. Oncotarget. 2017:9:7204-18.

41. Pereira SG, Moura J, Carvalho E, Empadinhas N. Microbiota of chronic diabetic wounds: ecology, impact, and potential for innovative treatment strategies. Front Microbiol. 2017;8. https://doi.org/10.3389/fmicb.2017.01791.

42. Jneid J, Lavigne JP, La Scola B, Cassir N. The diabetic foot microbiota: a review. Hum Microbiome J. 2017:5-6:1-6.

43. Gardiner M, Vicaretti M, Sparks J, Bansal S, Bush S, Liu M, et al. A longitudinal study of the diabetic skin and wound microbiome. PeerJ. 2017; 5. https://doi.org/10.7717/peerj.3543.

44. Malone M, Johani $\mathrm{K}$, Jensen SO, Gosbell IB, Dickson HG, Hu H, et al. Next generation DNA sequencing of tissues from infected diabetic foot ulcers. EBioMedicine. 2017:21:142-9.

45. MacDonald A, Brodell JD, Daiss JL, Schwarz EM, Oh I. Evidence of differentia microbiomes in healing versus non-healing diabetic foot ulcers prior to and following foot salvage therapy. J Orthop Res. 2019;37:1596-603.

46. Verbanic S, Shen Y, Lee J, Deacon JM, Chen IA. Microbial predictors of healing and short-term effect of debridement on the microbiome of chronic wounds. Npj Biofilms Microbiomes. 2020;6:1-11.

47. Sloan TJ, Turton JC, Tyson J, Musgrove A, Fleming VM, Lister MM, et al. Examining diabetic heel ulcers through an ecological lens: microbial community dynamics associated with healing and infection. J Med Microbiol. 2019;68:230-40.

48. Mottola C, Semedo-Lemsaddek T, Mendes JJ, Melo-Cristino J, Tavares L, Cavaco-Silva $P$, et al. Molecular typing, virulence traits and antimicrobial resistance of diabetic foot staphylococci. J Biomed Sci. 2016;23. https://doi. org/10.1186/s12929-016-0250-7.

49. Bowling FL, Jude EB, Boulton AJM. MRSA and diabetic foot wounds: contaminating or infecting organisms? Curr Diab Rep. 2009;9:440.

50. Zhao G, Usui ML, Underwood RA, Singh PK, James GA, Stewart PS, et al. Time course study of delayed wound healing in a biofilm-challenged diabetic mouse model. Wound Repair Regen. 2012;20:342-52.

51. Kong EF, Johnson JK, Jabra-Rizk MA. Community-associated methicillin-resistant Staphylococcus aureus: an enemy amidst us. PLOS Pathog. 2016;12:e1005837.

52. Shettigar K, Murali TS. Virulence factors and clonal diversity of Staphylococcus aureus in colonization and wound infection with emphasis on diabetic foot infection. Eur J Clin Microbiol Infect Dis. 2020. https://doi. org/10.1007/s10096-020-03984-8.

53. Stacy A, Everett J, Jorth P, Trivedi U, Rumbaugh KP, Whiteley M. Bacterial fight-and-flight responses enhance virulence in a polymicrobial infection. Proc Natl Acad Sci. 2014;111:7819-24.

54. Bowler PG, Duerden BI, Armstrong DG. Wound microbiology and associated approaches to wound management. Clin Microbiol Rev. 2001;14:244-69.

55. Park J-U, Oh B, Lee JP, Choi M-H, Lee M-J, Kim B-S. Influence of microbiota on diabetic foot wound in comparison with adjacent normal skin based on the clinical features. BioMed Research International. 2019. https://doi.org/10. 1155/2019/7459236.

56. Dalton T, Dowd SE, Wolcott RD, Sun Y, Watters C, Griswold JA, et al. An in vivo polymicrobial biofilm wound infection model to study interspecies interactions. PLOS ONE. 2011;6:e27317.

57. Pastar I, Nusbaum AG, Gil J, Patel SB, Chen J, Valdes J, et al. Interactions of methicillin resistant Staphylococcus aureus USA300 and Pseudomonas aeruginosa in polymicrobial wound infection. PLOS ONE. 2013;8. https://doi. org/10.1371/journal.pone.0056846.

58. Rosenbluth DB, Wilson K, Ferkol T, Schuster DP. Lung function decline in cystic fibrosis patients and timing for lung transplantation referral. Chest. 2004;126:412-9.

59. Hendricks KJ, Burd TA, Anglen JO, Simpson AW, Christensen GD, Gainor BJ. Synergy between Staphylococcus aureus and Pseudomonas aeruginosa in a rat model of complex orthopaedic wounds. JBJS. 2001;83:855-61.

60. DeLeon S, Clinton A, Fowler H, Everett J, Horswill AR, Rumbaugh KP. Synergistic interactions of Pseudomonas aeruginosa and Staphylococcus aureus in an in vitro wound model. Infect Immun. 2014;82:4718-28. 
61. Shankar EM, Mohan V, Premalatha G, Srinivasan RS, Usha AR. Bacterial etiology of diabetic foot infections in South India. Eur J Intern Med. 2005;16: $567-70$.

62. Abdulrazak A, Ibrahim Bitar Z, Ayesh Al-Shamali A, Ahmed Mobasher L. Bacteriological study of diabetic foot infections. J Diabetes Complications. 2005;19:138-41.

63. Sadeghpour Heravi F, Zakrzewski M, Vickery KG, Armstrong D, Hu H. Bacterial diversity of diabetic foot ulcers: current status and future prospectives. J Clin Med. 2019;8:1935.

64. Mastropaolo MD, Evans NP, Byrnes MK, Stevens AM, Robertson JL, Melville SB. Synergy in polymicrobial infections in a mouse model of type 2 diabetes. Infect Immun. 2005;73:6055-63.

65. Lipsky BA, Senneville É, Abbas ZG, Aragón-Sánchez J, Diggle M, Embil JM, et al. Guidelines on the diagnosis and treatment of foot infection in persons with diabetes (IWGDF 2019 update). Diabetes Metab Res Rev. 2020;36:e3280.

66. Uçkay I, Berli M, Sendi P, Lipsky BA. Principles and practice of antibiotic stewardship in the management of diabetic foot infections. Curr Opin Infect Dis. 2019;32:95-101.

67. Price LB, Liu CM, Melendez JH, Frankel YM, Engelthaler D, Aziz M, et al. Community analysis of chronic wound bacteria using 165 rRNA gene-based pyrosequencing: impact of diabetes and antibiotics on chronic wound microbiota. PLOS ONE. 2009:4:e6462.

68. Merkley MA, Bice TC, Grier A, Strohl AM, Man L-X, Gill SR. The effect of antibiotics on the microbiome in acute exacerbations of chronic rhinosinusitis. Int Forum Allergy Rhinol. 2015;5:884-93.

69. Mohseni S, Bayani M, Bahmani F, Tajabadi-Ebrahimi M, Bayani MA, Jafari P, et al. The beneficial effects of probiotic administration on wound healing and metabolic status in patients with diabetic foot ulcer: a randomized, double-blind, placebo-controlled trial. Diabetes Metab Res Rev. 2018;34: e2970.

70. Morozova W, Kozlova YN, Ganichev DA, Tikunova NV. Bacteriophage treatment of infected diabetic foot ulcers. In: Azeredo J, Sillankorva S, editors. Bacteriophage therapy: from lab to clinical practice. New York: Springer; 2018. p. 151-8. https://doi.org/10.1007/978-1-4939-7395-8_13.

71. Duplessis CA, Biswas B. A review of topical phage therapy for chronically infected wounds and preparations for a randomized adaptive clinical trial evaluating topical phage therapy in chronically infected diabetic foot ulcers. Antibiotics. 2020;9. https://doi.org/10.3390/antibiotics9070377.

72. Dunyach-Remy C, Cadière A, Richard J-L, Schuldiner S, Bayle S, Roig B, et al. Polymerase chain reaction-denaturing gradient gel electrophoresis (PCRDGGE): a promising tool to diagnose bacterial infections in diabetic foot ulcers. Diabetes Metab. 2014;40:476-80.

73. Smith K, Collier A, Townsend EM, O'Donnell LE, Bal AM, Butcher J, et al. One step closer to understanding the role of bacteria in diabetic foot ulcers: characterising the microbiome of ulcers. BMC Microbiol. 2016;16. https://doi. org/10.1186/s12866-016-0665-z.

74. Garg AX, Hackam D, Tonelli M. Systematic review and meta-analysis: when one study is just not enough. Clin J Am Soc Nephrol. 2008:3:253-60.

\section{Publisher's Note}

Springer Nature remains neutral with regard to jurisdictional claims in published maps and institutional affiliations.

Ready to submit your research? Choose BMC and benefit from:
- fast, convenient online submission
- thorough peer review by experienced researchers in your field
- rapid publication on acceptance
- support for research data, including large and complex data types
- gold Open Access which fosters wider collaboration and increased citations
- maximum visibility for your research: over 100M website views per year
At BMC, research is always in progress.
Learn more biomedcentral.com/submissions

